\title{
Can evolutionary dynamics explain free riding in experiments? *
}

\author{
John H. Miller \\ Santa Fe Institute, Santa Fe, NM 87501, USA \\ James Andreoni \\ University of Wisconsin-Madison, Madison, WI 53706, USA \\ Received 17 December 1990 \\ Accepted 11 February 1991
}

\begin{abstract}
Free riding experiments have generated many anomalous results that cannot be explained with standard Nash equilibrium models of public goods. This paper examines the experiments within the context of evolutionary game theory. This approach models the decision process of agents by an adaptive learning algorithm. The algorithm 'strengthens' strategies that do relatively well and 'weakens' strategies that do relatively poorly. This model produces predictions that are strikingly similar to those generated by experiments. Hence, evolutionary game theory may provide a way to understanding otherwise anomalous observations on contributions to public goods.
\end{abstract}

\section{Introduction}

Free riding experiments have generated many anomalous results that cannot be explained with standard Nash equilibrium models of public goods [see Dawes and Thaler (1988) for a review]. This paper examines these experiments within the context of evolutionary game theory. We model the decision process of agents by an adaptive learning algorithm. The algorithm 'strengthens' strategies that do relatively well and 'weakens' strategies that do relatively poorly. The model produces predictions that are strikingly similar to results generated in experiments. Hence, evolutionary dynamics may provide a way to understand the otherwise anomalous observations on contributions to public goods.

In the typical public good experiment, subjects are formed into groups of size $g$, and are given an exogenous incomes of $I$. Each subject must then allocate $I$ between a public and private good. After all individuals make their allocation decisions, each receives a payoff equal to

$$
p_{i}=I-c_{i}+m g \bar{c},
$$

where $c_{i}$ is subject $i$ 's contribution to the public good, $m$ is the known marginal per capita return from giving to the public good, and $\bar{c}$ is the average contribution to the public good. The parameter $m$ is chosen so that $0<m<1$ and $m g>1$. Given this design, $c_{i}=0$ (free riding) is the dominant

* Miller thanks the Santa Fe Institute's programs on Adaptive Computation for financial support, and Andreoni thanks the National Science Foundation, grant SES-8821204. We are also grateful to Robyn Dawes, Roy Gardner, R. Mark Isaac, Gerald Marwell, and James M. Walker for helpful comments. 
strategy Nash equilibrium, and $c_{i}=I$ is the symmetric Pareto efficient allocation. Values of $g$ typically range from 4 to $10, m$ ranges from 0.3 to 0.75 , and $I$ is usually under $\$ 1.00$. This design has been used by Marwell and Ames (1981), Isaac, Walker and Thomas (1984), Isaac, McCue and Plott (1985), Andreoni (1988b), Isaac and Walker (1988), and others.

There are several regularities in these experiments. Subjccts on average allocate about half of their income to the public good in the initial play of either a single-shot or repeated game, regardless of their group size or marginal return. In iterated games provision 'decays' toward the free riding equilibrium, although exact free riding behavior is seldom observed. Even more surprising results have been found recently by Isaac, Walker, and Williams (1990). Studying groups in size 4, 10, 40 and 100, they found that, as above, subjects tend to give about half of their endowment to public good in the first round with decay in later rounds, however, they also observed that the rate of decay is inversely related to group size - larger groups had slower rates of decay. In addition, they found that as the marginal return from contributing to the public good rises (even though the equilibrium is unchanged), decay markedly slows for all group sizes, and the paths of play became largely indistinguishable.

Existing models of rational Nash equilibrium play cannot explain any of these results. Current public goods theory indicates that free riding should be far more prevalent than is actually observed, and that cooperation should be more difficult to sustain in large groups [Andreoni (1988b)]. Although the equilibrium is the same in all models, $m$ and $g$ have systematic effects on behavior. In an effort to understand the above anomalies, we examine a simple model of decision making based on evolutionary dynamics. We find striking parallels between the dynamics generated by this model and the experimental findings. In particular, the model predicts the decay toward free riding, with slower decay for larger groups and for groups with higher marginal returns.

\section{An evolutionary model of decision making}

Economists have become increasingly interested in models of learning, adaptation, and evolution [c.g. Milgrom and Roberts (1990), Fudenberg and Maskin (1990), and Fudenberg and Kreps (1988)], and in using evolutionary game theory to examine experimental findings [Crawford (1990) and Andreoni and Miller (1990)]. The model used here is based on replicator dynamics [Schuster and Sigmond (1983)]. Applications of this dynamic by game theorists include Axelrod and Hamilton (1981), Samuelson (1988), Nachbar (1990), and Boylan (1990). Replicator dynamics are based on the notion that strategies that perform relatively well will replace those that perform relatively poorly.

Suppose there are $N$ types of strategies interacting in a population of fixed size. Each strategy of type $i$ always contributes $c_{i}$ dollars to the public good. Let $x_{i}^{l}$ be the proportion of type $i$ strategies in the population at time $t$. Then, we expected payoff to an agent using strategy type $i$ is

$$
p_{\imath}=I-c_{i}+m g \sum_{i=1}^{N} c_{j} x_{j}^{t}=I-c_{i}+m g \bar{c}^{t},
$$

where $\bar{c}^{t}$ is the average contribution at time $t$.

Replicator dynamics provide a natural algorithm for allowing the weights, $x_{i}^{t}$, to evolve over time. The dynamics are given by

$$
x_{i}^{t+1}=x_{i}^{t} \frac{p_{i}}{\sum_{j=1}^{N} p_{j} x_{j}^{t}}=x_{i}^{t} \frac{I-c_{i}+m g \bar{c}^{t}}{I-\bar{c}^{t}+m g \bar{c}^{t}} .
$$


This adjustment process captures the intuitive notion that strategies should be 'strengthened' if they do better than average, and 'weakened' if they do worse, and that the rate of adjustment should be faster the farther a strategy is from the mean. The dynamic also has the feature that a $\$ 1$ deviation from the mean payoff will result in a faster adjustment when the mean is $\$ 10$ than when it is $\$ 100$. This accords with the intuitive rule-of-thumb that $10 \%$ differences are more 'urgent' or 'costly' than $1 \%$ differences. Note also that strategy types that are not initially in the population can never emerge, and those that have support will never (in finite time) disappear.

There are several ways in which replicator dynamics may be viewed as a plausible model of decision making. First, agents may be playing an evolutionary game [see Maynard Smith and Price (1973), and van Damme (1989 ch. 9)]. Over time, better strategies proliferate by some adaptive selection process. In this case, strategies can be viewed as individuals whose presence is determined adaptively, or as 'ideas' that reside in the strategy space of players who choose moves randomly, as in a mixed strategy, using $x_{i}^{t}$ as weights. Second, agents may actually be concerned with their performance relative to the average. Several experiments [Andreoni (1990), and Kahneman and Knetsch (1990)] have demonstrated that the behavior of subjects is consistent with the hypothesis that people enjoy being part of cooperative outcomes, but that they are unwilling to have their cooperation exploited by other more selfish players [see Andreoni (1989) and Ledyard (1990) on 'warm glow' giving]. If this is true, then those who give above average amounts will always feel exploited, and hence will switch to more selfish strategies. Finally, the model mirrors prominent psychological theories of decision making based on 'reference points' [see Ableson and Levi (1985) for a revicw]. According to these theories, people adjust their decisions more rapidly the more the outcome falls short of some reference point, such as the average.

\section{Theoretical predictions}

Despite its simplicity, the model makes a number of predictions about the behavior of agents in the system. We will present six propositions. All of these propositions have rigorous proofs, although, for brevity, we will sometimes only outline the proofs. The propositions concern only those strategies that have positive support during the initial time period, and we assume that at least two such strategies exist.

Proposition 1. Any strategy that contributes less (more) than the average contribution will grow (decline) in proportion over time.

Proof. By eq. (1), $x_{i}^{t+1} / x_{i}^{t}>(=,<) 1$ as $c_{i}<(=,>) \bar{c}^{t}$.

Proposition 2. The average contribution will fall over time.

Proof. At any point in time, the weights on those $c_{i}\left\langle\bar{c}^{t}\right.$ increase, while the weights on those $\left.c_{i}\right\rangle \bar{c}^{t}$ decrease. Thus, the average must decline.

Proposition 3. The system will converge asymptotically to the strategy that does the most free riding.

Proof (intuition). Proposition 1 indicates that the growth always occurs in strategies that give less than the current average. Proposition 2 indicates that the average will decline over time. Thus, as the average moves down, the weights will eventually be concentrated on the strategy that gives the least to the public good. 
Propositions 1, 2, and 3 show that replicator dynamics will produce decay towards free riding. Over time, the more cooperative strategies will be replaced by those that give less to the public good. Proposition 3 implies that given sufficient time the entire population will use the strategy that does the most free riding.

Proposition 4. Starting from the same initial distribution, the dynamics will be identical for $m g=k$ where $k$ is a constant.

Proof. Equation (1) is identical for all systems in this case.

Proposition 5. As $m g$ increases, the rate of adjustment slows, and as $m g \rightarrow \infty$ then $x_{i}^{t+1} / x_{i}^{t} \rightarrow 1$.

Proof. It can be shown that the absolute value of $1-x_{i}^{t+1} / x_{i}^{t}$ decreases as $m g$ increases. For the latter statement, take the limit of eq. (1).

Proposition 6. As mg increases, the decline in average contributions slows.

Proof (intuition). As in the proof of Proposition 5, a larger $m g$ slows the changes in $x_{i}^{t+1}$ relative to $x_{i}^{t}$, and thus starting from the same initial conditions the convergence is slowed.

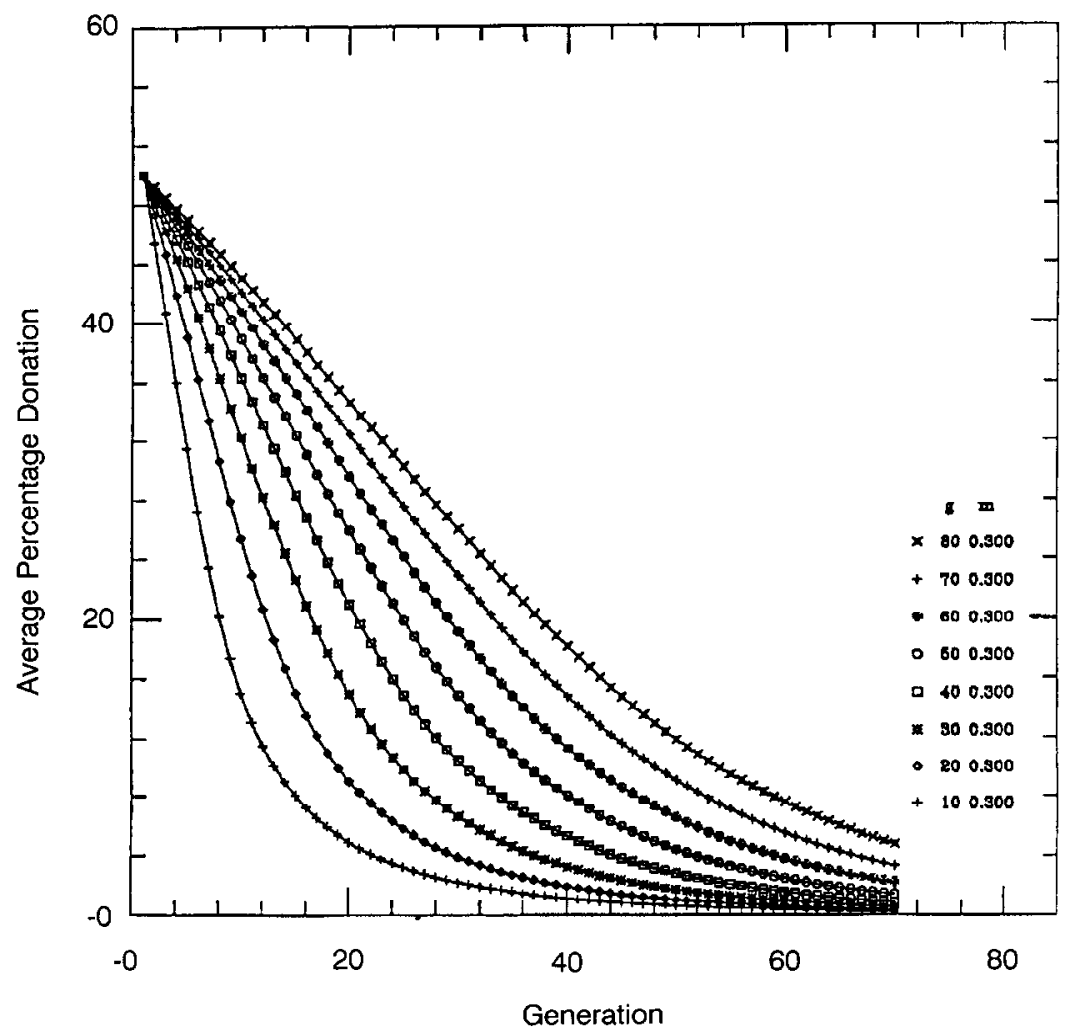

Fig. 1. Uniform initial distribution. 


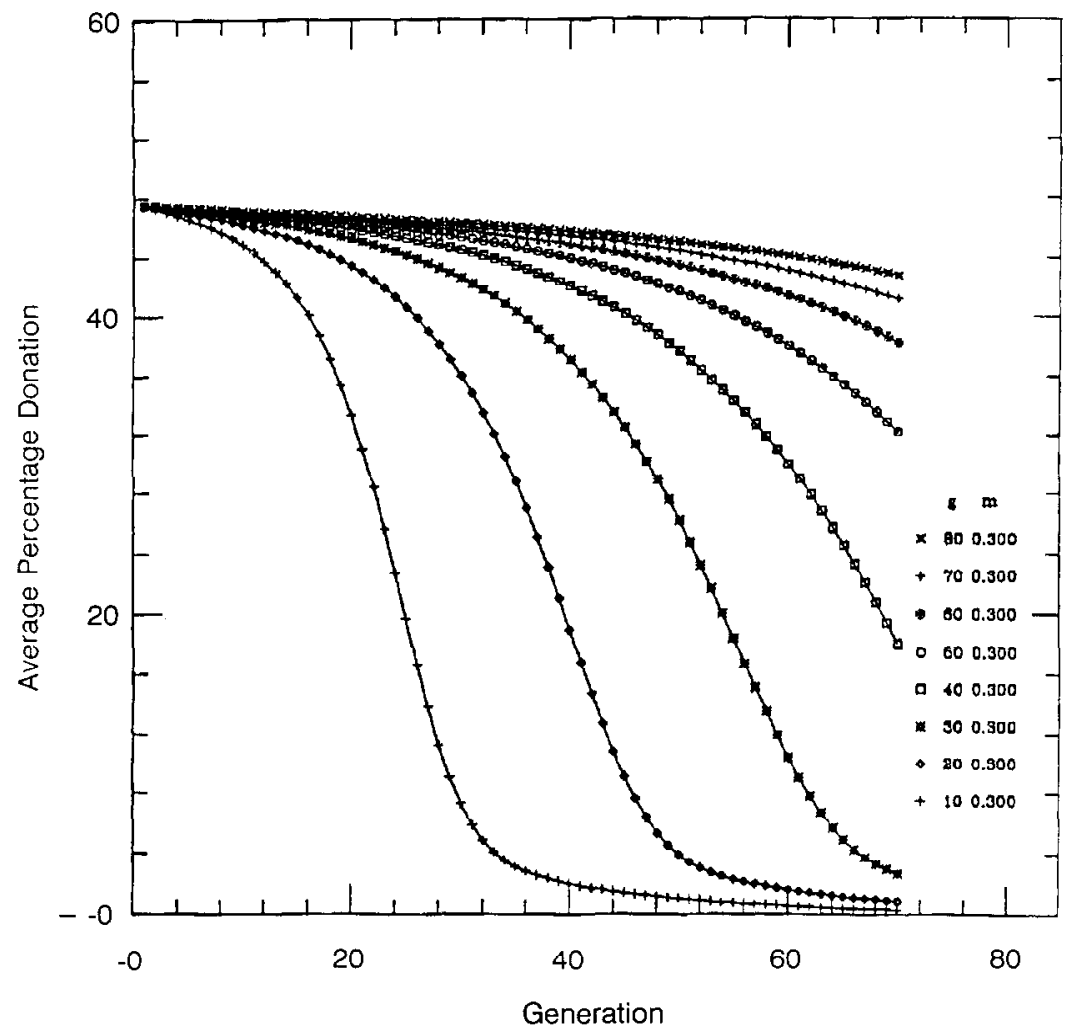

Fig. 2. Biased initial distribution.

Proposition 4, 5, and 6 indicate that decay should be slower the larger the group size, $g$, and the higher the marginal return $m$. For any average contribution $\bar{c}^{t}$, the higher $m g$ the higher the payoff of every subject. The higher the total payoff, the lower each person's percentage deviation from the mean, hence, the slower the rate of decay. Although the optimizing model would predict, for instance, that free riding will increase as $g$ increases, this model does not: Since all strategies perform about the same, there is a smaller incentive for any agent to change strategies. Hence, a behavioral model in which subjects choose their first move more or less randomly, and then adjust their behavior according to a rule like (1), will produce a dynamic path similar to the paths observed in experiments.

Figures 1 and 2 illustrate the above propositions. These figures are based on an initial population of 20 strategies distributed between giving $0 \%$ of $I$ to the public good and giving $100 \%$ of $I$. Figure 1 illustrates a uniform initial distribution of strategy types, and fig. 2 illustrates one that is concentrated around 50\%. ${ }^{1}$ The paths are shown for a variety of groups sizes, with $m=0.3$. Although the figures illustrate the dynamics over 70'generations' of play, this does not necessarily correspond to any particular time scale. The rates of adjustment can be quickened or slowed in the algorithm by, for example, adding a constant to the payoff values. This constant could even be estimated using experimental data. 


\section{Discussion and conclusion}

The replicator-dynamic model of evolutionary decision making has captured the anomalous results from experiments. Contributions will decay toward the dominant strategy Nash level, and the rate of decay will be slower for larger groups and for groups with higher marginal returns. While this model is very simple, more complex versions of evolutionary dynamics are likely to lead to similar results. For example, identical qualitative results hold if agents who perform worse than avcrage only switch to the average strategy, or if agents follow a complicated adaptive scheme, such as a genetic algorithm model [Holland (1975), Goldberg (1989)]. Although the simple replicator model discussed here does not have any explicit parameters, simple modifications could allow the speed of adjustment to be set parametrically.

There is one regularity found by Isaac and Walker (1988) and Isaac, Walker and Williams (1990) that is not yet captured by this simple model of decision making. Comparing separate groups in which $m g$ is identical, they find that decay is slower for groups with higher $m$ and lower $g$, than in groups with lower $m$ and higher $g$. While the difference is not vast, it is persistent. This is clearly at odds with proposition 4 above, which indicates that the dynamics depend only on $m g$. Hence, there may be additional benefits to more research into the evolutionary models of giving. For instance, the above model is based on the assumption that large and small groups sample the population at the same rate, that is, the dynamic is based on the population average, regardless of group size. However, subjects in small groups have less exposure to different 'types' of strategies, hence sample the population at a slower rate. As a result, their convergence to free riding may be slower, even though $m g$ is the same. Generalizing and developing this aspect of the model may yield predictions consistent with this last experimental result.

In conclusion, the simple evolutionary model of behavior is intuitively plausible, and can explain otherwise anomalous behavior. This suggests that our understanding of the private provision of public goods may be improved by more careful research into evolutionary game theory, and by theory and experiments that examine the motives, decision processes, and dynamics of public goods games.

\section{References}

Ableson, Robert and Ariel Levi, 1985, Decision making and decision theory, The handbook of social psychology, Vol. III (Random House Press, New York).

Andreoni, James, 1988a. Privately provided public goods in large economies: The limits of altruism, Journal of Public Economics, 35, 57-73.

Andreoni, James, 1988b, Why free ride? Strategies and learning in public good experiments, Iournal of Public Economics 37 , 291-304.

Andreoni, James, 1989, Giving with impure altruism: Applications to charity and Ricardian equivalence, Journal of Political Economy 97, 1447-1458.

Andreoni, James, 1990, An experimental test of the public goods crowding-out hypothesis, Working paper 9006 (University of Wisconsin, Madison, WI).

Andreoni, James and John H. Miller, 1990, Auctions with adaptive artificially intelligent agents, Working paper (Santa Fe Institute, Santa Fe, NM).

Axelrod, Robert and William D. Hamilton, 1981, The evolution of cooperation, Science 211, 1390-1398.

Boylan, Richard, 1990. Evolutionary equilibria with mutation, Working paper (California Institute of Technology, Passadena, CA).

Crawford, Vincent P., 1989, An 'evolutionary' explanation of Van Huyck, Battalio, and Beil's experimental results on coordination, Working paper (University of California, San Diego, CA).

Dawes, Robyn and Richard Thaler, 1988, Anomalies: cooperation, Journal of Economic Perspectives 2, $187-198$. 
Fudenberg, Drew and David Kreps, 1988, A theory of learning, experimentation, and equilibrium in games, Mimeo. (M.I.T., Cambridge, MA).

Fudenberg, Drew and Eric Maskin, 1990, Evolution and cooperation in noisy repeated games. American Economic Review, forthcoming.

Goldberg, David, 1989, Genetic algorithms in search, optimization and machine learning (Addison-Wesley, New York).

Holland, John, 1975, Adaptation in natural and artificial systems (University of Michigan Press, Ann Arbor, MI).

Isaac, R. Mark, Kenneth F. McCue, and Charles, R. Plott, 1985, Public goods provision in an experimental environment, Journal of Public Economics, 26, 51-74.

Isaac, R. Mark and James M. Walker, 1988, Group size effects in public goods provision: The voluntary contribution mechanism, Quarterly Journal of Economics 53, 179-200.

Isaac. R. Mark, James M. Walker, and Susan H. Thomas, 1984, Divergent evidence on free riding: An experimental examination of possible explanations, Public Choice 43, 113-149.

Isaac, R. Mark, James M. Walker and Arlington Williams, 1990, Group size and the voluntary provision of public goods: Experimental evidence utilizing very large groups, Mimeo (University of Arizona, Tucson, AZ).

Kahneman, Daniel and Jack Knetsch, 1990, Valuing public goods: The purchase of moral satisfaction, Working paper (University of California, Berkeley, CA).

Kim, Oliver and Mark Walker, 1984, The free rider problem: Experimental evidence, Public Choice 43, 3-24.

Ledyard, John O., 1990, Is there a problem with public good provision?, Working paper (California Institute of Technology, Passadena, CA).

Marwell, Gerald and Ruth El. Ames, 1981, Economists free ride, does anyone else?, Journal of Public Economics 15, 295-310. Maynard Smith, John and G.R. Price, 1973, The logic of animal conflicts, Nature 246, 15-18.

Milgrom, Paul R. and John Roberts, 1990, Adaptive and sophisticated learning in repeated normal form games. Mimeo. (Stanford University, Stanford, CA).

Nachbar, John H., 1990, An ecological approach to economic games, International Journal of Game Theory, forthcoming. Samuelson, Larry, 1988, Evolutionary foundations of solution concepts for finite, two-player, normal-form, games, in: M.Y. Vardi, ed., Theoretical aspects of reasoning about knowledge, (Morgan Kaufmann, Los Altos, CA) 211-225.

Schuster, Peter and Karl Sigmund, 1983, Replicator dynamics, Journal of Theoretical Biology 100, 533-538.

van Damme. Eric, Stability and perfection in Nash equilibria (Springer-Verlag, New York). 